\title{
Double Higgs production at the LHC
}

\section{Louis Portales*, for the ATLAS and CMS Collaborations}

Laboratoire Leprince-Ringuet, CNRS/Ecole Polytechnique

E-mail: louis.portales@cern.ch

An overview of the latest results on resonant and non-resonant Higgs boson pair production, using up to $140 \mathrm{fb}^{-1}$ of $\sqrt{s}=13 \mathrm{TeV}$ proton-proton collision data recorded during Run 2 of the LHC by the ATLAS and CMS Collaborations, is presented. A wide range of decay modes is covered, with one Higgs boson decaying to $b \bar{b}$, and the second to $b \bar{b}, W^{+} W^{-}, Z Z, \tau^{+} \tau^{-}$or $\gamma \gamma$, as well as different topologies, with studies of both resolved and boosted signals and for both gluon-gluon and vector-boson fusion production modes. Analysis improvements, and newly introduced techniques with respect to previous studies, are highlighted.

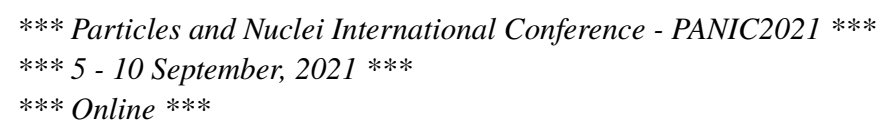

${ }^{*}$ Speaker 


\section{Introduction}

Since the discovery of the Higgs boson in 2012 [1-3], the associated production of pairs of Higgs bosons $(H H)$ has been one of the most sought after processes for the ATLAS [4] and CMS [5] Collaborations, for the insight it can provide on the Electroweak Symmetry Breaking Mechanism. HH pairs can be produced either through non-resonant or resonant processes. The non-resonant production mainly occurs through gluon-gluon fusion $(g g \mathrm{~F})$, with a low production cross-section of $\sigma_{\mathrm{SM}}^{\mathrm{NNLO}}(g g \mathrm{~F} H H)=31.1 \mathrm{fb}^{-1}$. The subleading production mechanism, the Vector-Boson Fusion (VBF), is also interesting to consider. Diagrams corresponding to the two production modes are shown in Figure 1. Although its production cross-section, $\sigma_{\mathrm{SM}}^{\mathrm{N}^{3} \mathrm{LO}}=1.73 \mathrm{fb}^{-1}$, is much lower than that of the $g g \mathrm{~F}$ production mode, its study has already allowed the production of a variety of interesting results. The study of non-resonant $H H$ production can provide insights on the Electroweak symmetry breaking mechanism, through the direct access to the trilinear Higgs coupling $\lambda_{H H H}$ and give an additional handle for the measurement of couplings of other particles to the Higgs boson. The resonant $H H$ production can also be a direct sign of new physics, with many BSM models predicting heavy spin-0 or spin-2 resonances $X$ decaying to $H H$ with significant branching ratios for $m_{X}>2 m_{H}$.
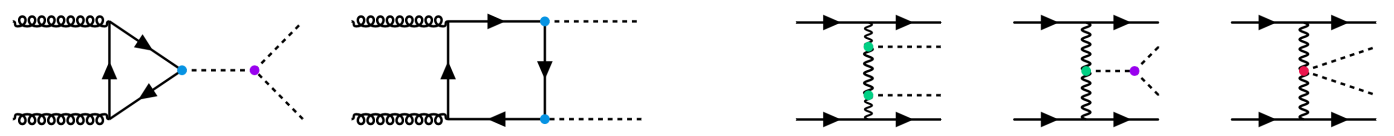

Figure 1: Main Feynman diagrams for the non-resonant production of Higgs boson pairs.

The choice of the final state used to study $H H$ production is mainly driven by the branching ratios (BR). So far, analyses have been looking at final states where at least one of the Higgs bosons decays to $b \bar{b}$, to benefit from the sizable $\operatorname{BR}(H \rightarrow b \bar{b}) \simeq 58 \%$, with the second Higgs decay selected accounting for the trade-off between the BR and the expected contamination from background processes.

Constraints on the $H H$ production cross-section have been set by the ATLAS and CMS collaborations using $36 \mathrm{fb}^{-1}$ of data collected in 2016 by the ATLAS and CMS Collaborations [6, 7], achieving upper limits on the $H H$ production cross-section of about ten times the SM prediction, from combinations of their respective analyses. These results were extrapolated to evaluate the expected constraints on the cross-section and on the trilinear Higgs coupling modifier $\kappa_{\lambda}$ at HL-LHC [8]. They highlight a possible exclusion of $\kappa_{\lambda}=0$ at a $95 \%$ confidence level from the combination of ATLAS and CMS results, but the experiments may not be able to claim the observation of the process, with the expected discovery significance from such a combination expected to reach $4.0 \sigma$.

The Run 2 of the LHC came to an end in 2018, and allowed both experiments to collect about $140 \mathrm{fb}^{-1}$ of data, with which numerous results on the topic of $H H$ have been made public. Most of them present significant improvements with respect to the partial Run 2 analyses. These analyses are reviewed in Section 25, covering all relevant published results as of September 2021, grouped with respect to the studied final states.

\section{2. $H H \rightarrow b b b b$ analyses}

A study of the non-resonant $H H \rightarrow b b b b$ production with a fully resolved final state is made by the CMS Collaboration [9], targetting both $\mathrm{VBF}$ and $g g \mathrm{~F}$ production modes. Events are required to contain at least 4 jets, and at least 3 of them are required to be well-identified b-jets. The b-jet pairing combinatorics issue is solved by requiring the two pairs of b-jet to minimize $d=\frac{\left|m_{H_{1}}-k m_{H_{2}}\right|}{\left(1+k^{2}\right)}$, where $k=1.04$ is the ratio of the two reconstructed Higgs bosons expected mass values, and $m_{H_{1}}$ and $m_{H_{2}}$ their respective reconstructed mass. Two event categories are defined for the VBF or $g g$ F signals, based on the identification of the two energetic forward jets characterizing VBF events, in addition to a dedicated BDT discriminant trained to separate the two signals. A second BDT discriminant is used for the signal extraction fit in the $g g \mathrm{~F}$ category, while in the VBF category, the di-Higgs invariant mass $m_{H H}$ is used instead. Limits are extracted for the coupling 
modifiers $\kappa_{\lambda}$ and $\kappa_{2 V}$, and found to be $-2.3<\kappa_{\lambda}<9.4$ (exp. $-5.0<\kappa_{\lambda}<12.0$ ) and $-0.1<\kappa_{2 V}<2.2$ (exp. $\left.0.4<\kappa_{2 V}<2.5\right)$. An upper limit on the $g g \mathrm{~F}+\mathrm{VBF}$ production cross-section is also set, and documented in Figure 2 (left), representing a five-fold improvement with respect to the partial Run-2 analysis.

The ATLAS Collaboration published an analysis targetting the VBF production mode specifically [10], based on their partial Run $2 \mathrm{ggF} \mathrm{HH} \rightarrow b b b b$ search [13], and adding to it the requirement of having to opposite- $\eta$ VBF jets. The analysis is further improved by introducing a BDT-based energy regression for b-jets, significantly improving the $b \bar{b}$ mass resolution. Both resonant and non-resonant production modes are probed from fits on the $m_{H H}$ spectrum. Limits on the resonant $\mathrm{HH}$ production are set for resonant mass hypotheses ranging from $250 \mathrm{GeV}$ to $1 \mathrm{TeV}$, with no significant excess found. The non-resonant production mode is used to set limits on $\kappa_{2 V}$, and the limits are set to $-0.43<\kappa_{2 V}<2.56$ (exp. $0.55<\kappa_{2 V}<2.72$ ).

Another VBF analysis is made public by the CMS Collaboration [14], that chooses to explore boosted topologies, where the decay products of the Higgs bosons are reconstructed as merged objects, to constrain $\kappa_{2 V}$, profiting from the expected increase in cross-section at high $m_{H H}$ for values of $\kappa_{2 V}$ different from one [15]. The two final state b-jet pairs are reconstructed as large-radius $(\mathrm{R}=0.8)$ anti- $k_{\mathrm{T}}$ jets. ParticleNet [16], a multiclass Graph neural network trained to identify boosted resonances decaying into $b \bar{b}, c \bar{c}$ or light-quark jets, is used to define purity-based signal categories, as well as a regressor for $m_{b b}$. Limits on both $\kappa_{V}$ and $\kappa_{2 V}$ are extracted from a combined fit to the $m_{H H}$ distributions in the three categories, and found to be $0.8<\kappa_{V}<1.2$ (exp. $\left.0.8<\kappa_{V}<1.2\right)$ and $0.6<\kappa_{2 V}<1.4$ (exp. $0.6<\kappa_{2 V}<1.4$ ) at $95 \%$ CL, excluding for the first time $\kappa_{2 V} \leq 0$ at more than $99.99 \% \mathrm{CL}$ assuming SM values of all other couplings involved.

Both ATLAS and CMS published an analysis dedicated to the search for resonant $H H$ production $[17,18]$. CMS focuses on the (semi-)boosted regime, looking for events with either two $\mathrm{R}=0.8$ jets or an $\mathrm{R}=0.8$ jet and two b-tagged $\mathrm{R}=0.4$ jets, to probe high resonance mass hypotheses $\left(m_{X}>1 \mathrm{TeV}\right)$. DeepAK8 [19], a discriminant built from convolutional neural networks, is used for the identification of the $H \rightarrow b \bar{b}$ candidates and for event categorization. A 2D histogram is used for signal extraction, defined from the mass of the leading $\mathrm{R}=0.8$ jet $m_{\mathrm{J} 1}$ and the reduced di-Higgs mass $\left.m_{\mathrm{red}}=m_{H H}-\left(m_{\mathrm{J} 1}-m_{H}\right)-\left(m_{\mathrm{J} 2}-m_{H}\right)\right)$, where $m_{H}=125.09 \mathrm{GeV}$. In the analysis from ATLAS, both resolved and boosted topologies are considered, and used to target different ranges of $m_{H H}$. A resolved channel is used to probe low resonance mass hypotheses, in which four b-tagged $\mathrm{R}=0.4$ jets are required, a BDT discriminant is used to find the best $\mathrm{b}$-jet pair combination, and an elliptical cut in the Higgs boson candidates mass plane is applied to define the signal region. In the boosted channel, used to probe high mass hypotheses, two $\mathrm{R}=1.0$ jets are reconstructed, with at least one of them required to be b-tagged, and events are categorized according to the number of b-tagged sub-jets found within the two large-R jets. Limits on resonant $H H$ production are set using $m_{H H}$ in both the resolved and boosted channels, as illustrated in Figure 2 (right). Similar sensitivities are achieved in both ATLAS and CMS analyses, and no significant excess over the SM background prediction is found. The largest deviation is found by ATLAS for $m_{X}=1.1 \mathrm{TeV}$ with a local significance of $2.6 \sigma(2.7 \sigma)$, for a spin-0(2) resonance, reduced to $1.0 \sigma(1.2 \sigma)$ when correcting for the look-elsewhere effect.

\section{3. $H H \rightarrow b b \tau \tau$ analyses}

In its resolved $\mathrm{HH} \rightarrow b b \tau \tau$ search [20], the ATLAS Collaboration uses events in which one of the final state $\tau$ leptons decays hadronically ( $\tau_{\text {had }}$ ), and the second one either hadronically or into a lighter charged lepton and two neutrinos $\left(\tau_{e}\right.$ or $\left.\tau_{\mu}\right)$, covering a cumulated $88 \%$ of all the possible decay combinations.

The analysis relies on the $\tau$ lepton decay modes to define three signal categories. Events in which a $\tau_{\text {had }} \tau_{\text {had }}$ pair is found, and is firing either a single- $\tau_{\text {had }}$ or di- $\tau_{\text {had }}$ trigger, are defining the first category. A second category (SLT) regroups $\tau_{e} \tau_{\text {had }}$ and $\tau_{\mu} \tau_{\text {had }}$ events firing single-lepton ( $e$ or $\left.\mu\right)$ triggers, while the third category (LTT) contains any $\tau_{\text {lep }} \tau_{\text {had }}$ events not entering the SLT category and firing a lepton $+\tau_{\text {had }}$ trigger.

The multijet and hadronic $t \bar{t}$ backgrounds, that can contain jets faking $\tau_{\text {had }}$, are estimated in a fully data-driven way. Background processes involving true $\tau$ leptons, the (semi-)leptonic $t \bar{t}$ production and $Z$ production associated with $b$ - or $c$-jets, have only their normalization corrected from data in control regions.

The analysis provides results for both non-resonant and resonant production. The non-resonant signal is extracted from a combined fit of a BDT discriminant in the $\tau_{\text {had }} \tau_{\text {had }}$ category, and DNNs in the SLT and 
LTT categories. The upper limit set on the $H H$ production cross-section, documented in Figure 2 (centre), presents a factor three improvement with respect to the partial Run-2 limit, and is, to date, the most stringent limit obtained from a single analysis. For the resonant signal constraints, Parametrized Neural Networks (as a function of $m_{H H}$ ) are trained in each category, and used in the signal extraction fits. Upper limits are determined as a function of the resonance mass, between $250 \mathrm{GeV}$ and $1.6 \mathrm{TeV}$, as shown in Figure 2 (right). The larger reported excess is found at $1 \mathrm{TeV}$, with a local (global) significance of $3.1 \sigma(2.1 \sigma)$.

ATLAS has also been investigating boosted topologies for the $b b \tau_{\text {had }} \tau_{\text {had }}$ final state [21], and extracted limits for mass hypotheses ranging from $800 \mathrm{GeV}$ to $3 \mathrm{TeV}$, with no significant excess found.

\section{4. $H H \rightarrow b b \gamma \gamma$ analyses}

The $H H \rightarrow b b \gamma \gamma$ final state is investigated by both ATLAS [22] and CMS [23]. This final state suffers from a very small BR $(\sim 0.26 \%)$. However, it highly benefits from its fully reconstructed final state, as well as from the excellent $m_{\gamma \gamma}$ resolution and relatively low background contamination.

The CMS analysis focuses on the non-resonant $g g \mathrm{~F}+\mathrm{VBF}$ production. It implements a very fine categorization based on BDT discriminants and on the reduced mass $m_{\text {red }}=m_{b b \gamma \gamma}-\left(m_{\gamma \gamma}-m_{H}\right)-\left(m_{b b}-\right.$ $m_{H}$ ), for a total of $12 \mathrm{ggF}$, and $2 \mathrm{VBF}$ signal regions. Limits on the VBF and $g g \mathrm{~F}+\mathrm{VBF}$ production cross-section, $\kappa_{\lambda}$ and $\kappa_{2 V}$ are extracted from a combined 2D fit of $m_{\gamma \gamma}$ and $m_{j j}$ over the 14 regions. Upper limits are set for the $g g \mathrm{~F}+\mathrm{VBF}$ and $\mathrm{VBF}$ production cross-section. The limit for $g g \mathrm{~F}+\mathrm{VBF}$ production, documented in Figure 2 (left) brings a factor four improvement compared to the partial Run 2 analysis. The coupling modifier $\kappa_{\lambda}$ is constrained within $-3.3<\kappa_{\lambda}<8.5$ (exp. $-2.5<\kappa_{\lambda}<8.2$ ), and $\kappa_{2} V$ within $-1.3<\kappa_{2 V}<3.5$ (exp. $-0.9<\kappa_{2 V}<3.1$ ).

A similar analysis strategy is employed by ATLAS, with a coarser categorization, but is used to search for both resonant and non-resonant production modes. For the non-resonant production, two categories, high-mass and low-mass, are defined, in each of which a dedicated BDT discriminant is trained and used for further categorization. For the resonant production, a mass-independent BDT is used for the categorization. In both cases, $m_{\gamma \gamma}$ is used for the signal extraction. The non-resonant search provides an upper limit on the $H H$ production cross-section of 4.1 (exp. 5.5) times the SM prediction, and constrains $\kappa_{\lambda}$ to be within $-1.5<\kappa_{\lambda}<6.7$ (exp. $-2.4<\kappa_{\lambda}<7.7$ ). Upper limits are set on the resonant production cross-section for $250 \leq m_{X} \leq 1000 \mathrm{GeV}$, shown in Figure 2 (right), with no significant excess found.

\section{5. $H H \rightarrow b b V V$ analyses}

The very first di-Higgs result made public by the CMS collaboration is the $H H \rightarrow b b 4 \ell[24](\ell=e, \mu)$, which is also the first public result focusing on that final state. This final state suffers from an even smaller BR than $H H \rightarrow b b \gamma \gamma(0.014 \%)$, but also benefits from its very clear final state signature, and from very small contamination from background, with the leading contribution coming from single-Higgs production. The analysis sets an upper limit on the $g g \mathrm{~F} \mathrm{HH}$ production cross-section, documented in Figure 2 (left), and $\kappa_{\lambda}$ is constrained within $-9<\kappa_{\lambda}<4$ (exp. $\left.-11<\kappa_{\lambda}<16\right)$.

A search for non-resonant $H H$ production in the $b b \ell v \ell v$ is performed by the ATLAS Collaboration [25]. The analysis is optimized for the $b b W W^{*}$ final state, but includes $b b Z Z^{*}(Z Z \rightarrow \ell \ell v v)$, and $b b \tau_{\text {lep }} \tau_{\text {lep }}$ in the signal definition. Two flavour-based categories are defined. A multi-class DNN is used for the event selection, separating the signal from the main backgrounds, $t \bar{t}, Z \rightarrow \ell \ell$ or $Z \rightarrow \tau \tau$. Cuts are applied around the $m_{\ell \ell}$ and $m_{b b}$ masses to define the signal regions, and the signal is extracted through a combined counting experiment across the analysis regions. An upper limit on the $g g \mathrm{~F} H H$ cross-section, as documented in Figure 2 (centre).

CMS used the $b b W W$ final state to probe the resonant $H H$ production at high resonance mass $(1<$ $\left.m_{X}<4.5 \mathrm{TeV}\right)$, by focusing on boosted topologies. The $H \rightarrow b b$ candidate is reconstructed as an $\mathrm{R}=0.8$ jet, and identified using DeepAK8. The analysis looks into semi-leptonic $H \rightarrow W W$ final states $(W W \rightarrow \ell v q q)$, with a single lepton and an $\mathrm{R}=0.8$ jet, and adds, with respect to the partial Run 2 analysis, fully-leptonic final states to improve the sensitivity to signal. A total of eight single-lepton and four di-lepton signal categories 
are defined, based on the object flavours, and DeepAK8 score and substructure information of the large-radius jets. Limits on the resonant production are set from a $2 \mathrm{D}$ fit to the $m_{b b}$ and $m_{H H}$ distributions. While no significant excess is found, one of the highlights of the result is the ten-folds improvement of the limits achieved with respect to the partial Run 2 analysis.

\section{Conclusion}

A wide selection of new di-Higgs results using the full Run 2 data have been made public by the ATLAS and CMS experiments. These results cover both the non-resonant and resonant, $g g \mathrm{~F}$ and VBF production modes, in either resolved or boosted final state topologies, and are found to yield unprecedented sensitivities, as shown in Figure 2. The limits set on cross-sections improved by a factor 3-to-10 with respect to the previously published analyses, with single analyses reaching sensitivity levels comparable to the partial Run 2 analyses combinations, raising the hopes for an observation of the $H H$ production at HL-LHC.
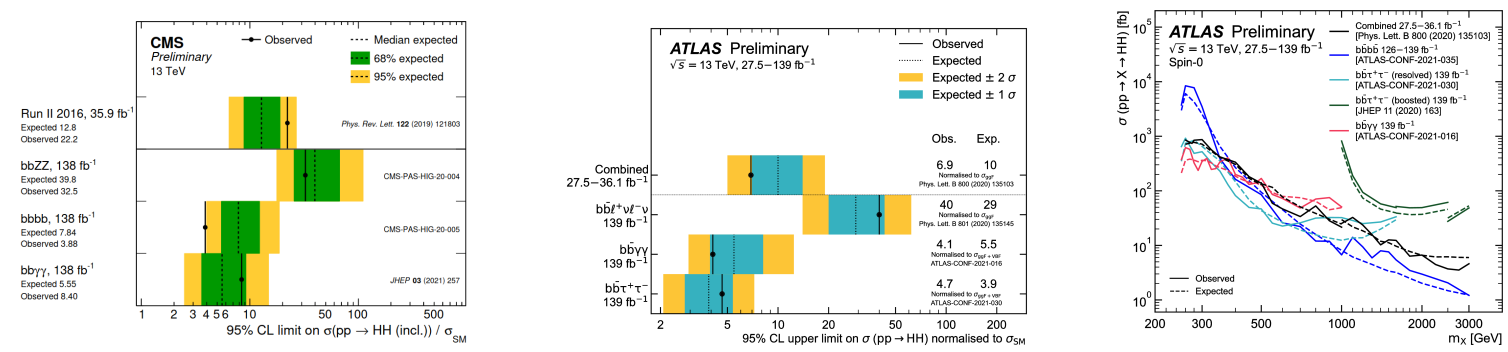

Figure 2: Summary plots for the non-resonant $H H$ cross-section upper limits set by the CMS [11] (left) and ATLAS [12] (centre) Collaborations, and resonant $H H$ cross-section upper limits as a function of the resonance mass set by ATLAS [12] (right). Results for the partial Run 2 combinations are displayed on each figure for comparison.

\section{References}

[1] ATLAS Collaboration, Phys. Lett. B 716 (2012) 1, doi:10.1016/jphysletb.2012.08.020

[2] CMS Collaboration, Phys. Lett. B 716 (2012) 30, doi:10.1016/jphysletb.2012.08.021

[3] CMS Collaboration, JHEP 06 (2013) 081, doi:10.1007/JHEP06(2013)081

[4] ATLAS Collaboration, JINST 3 S08003 (2008), doi:10.1088/1748-0221/3/08/S08003

[5] CMS Collaboration, JINST 3 S08004 (2008), doi:10.1088/1748-0221/3/08/S08004

[6] ATLAS Collaboration, Phys. Lett. B 800 (2020) 135103, doi:10.1016/j.physletb.2019.135103.

[7] CMS Collaboration, Phys. Rev. Lett. 122 (12) (2019) 121803, doi:10.1103/PhysRevLett.122.121803.

[8] HL-LHC Working Group, arXiv:1902.00134

[9] CMS Collaboration, CMS Physics Analysis Summary CMS-PAS-HIG-20-005.

[10] ATLAS Collaboration, JHEP 07 (2020) 108, doi:10.1007/JHEP07(2020)108.

[11] CMS Collaboration, Higgs PAG Summary Plots

[12] ATLAS Collaboration, ATLAS PUB Note ATL-PHYS-PUB-2021-031

[13] ATLAS Collaboration, JHEP 01 (2019) 030, doi:10.1007:JHEP01(2019)030.

[14] CMS Collaboration, CMS Physics Analysis Summary CMS-PAS-B2G-21-001.

[15] F.Bishara, R.Contino and J.Rojo, Eur. Phys. J. C (2017) 77:481, doi:10.1140/epjc/s10052-017-5037-9.

[16] H.Qu and L.Gouskos, Phys. Rev. D 101 (2020) 056019, doi:10.1103/PhysRevD.101.056019.

[17] ATLAS Collaboration, ATLAS Conference Note ATLAS-CONF-2021-035.

[18] CMS Collaboration, CMS Physics Analysis Summary CMS-PAS-B2G-20-004.

[19] CMS Collaboration, JINST 15 (2020) P06005, doi:10.1088/1748-0221/15/06/P06005.

[20] ATLAS Collaboration, ATLAS Conference Note ATLAS-CONF-2021-030.

[21] ATLAS Collaboration, JHEP 11 (2020) 163, doi:10.1007/JHEP11(2020)163.

[22] ATLAS Collaboration, ATLAS Conference Note ATLAS-CONF-2021-016.

[23] CMS Collaboration, JHEP 03 (2021) 257, doi:10.1007/JHEP03(2021)257.

[24] CMS Collaboration, CMS Physics Analysis Summary CMS-PAS-HIG-20-004.

[25] ATLAS Collaboration, Phys. Lett. B 801 (2020) 135145, doi:10.1016/j.physletb.2019.135145.

[26] CMS Collaboration, CMS Physics Analysis Summary CMS-PAS-B2G-20-007. 